\title{
Análisis de la aplicación de la Flexibilización Laboral en el Ecuador como estrategia para incrementar el empleo
}

\section{Analysis of the application of Labor Flexibility in Ecuador as a strategy to increase employment}

\author{
Martha Cecilia Vallejo Luzuriaga ${ }^{1 *}$ y Margarita Graciela Ayala Bolaños ${ }^{1}$ \\ ${ }^{1}$ Universidad del Pacifico \\ *matha.vallejo@upacifico.edu.ec
}

DOI: https://doi.org/10.26871/killkana_social.v2i4.374

\begin{abstract}
Resumen
Las reformas en materia laboral son una necesidad inminente en tiempos de crisis, el Ecuador finalmente es víctima de una crisis económica global y los más afectados son los sectores productivos, las reformas laborales buscan básicamente aumentar los niveles de empleo, la estabilidad laboral y estar en mejores condiciones de competir ante el resto de economías sobre todo los estados vecinos. En diversos países en el mundo, en especial América Latina y el Ecuador llevan a cabo reformas laborales con el objetivo de bajar las altas tasas de desempleo, impulsar la competitividad del aparato productivo, hacer mejor uso de la fuerza laboral y dotar a la economía de mayor dinamismo; sin embargo, los resultados siguen siendo desalentadores. Y es que cuando se habla de la flexibilización laboral, pues existen concepciones marcadamente diferentes en torno a su significado y esto hace que los sectores de la economía no lleguen a un acuerdo. Asimismo, es importante señalar que existen numerosas medidas económicas, las cuales pueden incidir sobre el mercado laboral. Este artículo agrupa diferentes posturas sobre la flexibilización laboral y su incidencia en la economía, la empresa y las condiciones de vida de los trabajadores.
\end{abstract}

Palabras clave: Flexibilización laboral, estabilidad laboral, desempleo, fuerza laboral.

\begin{abstract}
Labor reforms are an imminent need in times of crisis, Ecuador is finally the victim of a global economic crisis and the most affected are the productive sectors, labor reforms basically seek to increase employment levels, job stability and be in Better conditions to compete with the rest of the economies, especially the neighboring ones. Several countries in the world, especially Latin America and Ecuador, carry out labor reforms aimed at lowering high rates of unemployment, boosting the competitiveness of the productive apparatus, making better use of the labor force and giving the economy greater dynamism; However, the results remain daunting. And is that when talking about labor flexibility, because there are markedly different conceptions around its meaning and this makes the sectors of the economy do not reach an agreement. It is also important to note that there are numerous economic measures, which may affect the labor market. This article groups different positions on labor flexibility and its impact on the economy, business and the living conditions of workers.
\end{abstract}

Key words: Labor flexibility, job stability, unemployment, labor force.

\section{Introducción}

La modernización de la legislación laboral ha sido la causal para favorecer el ingreso de hombres y mujeres en el mercado laboral, se esperaba que sirva de dinamizador para reducir uno de los problemas que tiene el Ecuador que es el desempleo.

La flexibilización del empleo es un mecanismo que elimina la forma tradicional de concebir el trabajo. También permite que las empresas adecuen el número de trabajadores contratados o los cambios de su nivel y actividad, al facilitar y reducir el despido por causas económicas y tecnológicas.

Con la flexibilización del empleo se podría registrar los números de contratados en la economía y reducir los costos laborales no salariales como incentivos para la formalización de los contratos de trabajo.

En una economía globalizada y cambiante por efectos tecnológicos, las empresas tienen que ser flexibles al estructurar sus puestos de trabajo, contribuyendo a mejorar el problema que atraviesa actualmente el país.

La flexibilización laboral vuelve al debate, esta busca facilitar a las empresas la contratación de nuevos emplea- 
dos, por períodos de tiempo cortos y con procedimientos sencillos de desvinculación. La flexibilización laboral podría favorecer a la disminución del desempleo y con los mecanismos adecuados lograr mantener la estabilidad laboral tan anhelada por todas las personas.

Esta investigación es de tipo básica que se fundamenta en el análisis de la información obtenida de organismos nacionales e internacionales, así como de fuente primaria. Es una investigación de tipo descriptivo y cualitativa, de tipo educativa que busca informar al lector sobre ventajas y desventajas de la flexibilización laboral. La investigación compara diversas situaciones dadas en materia de flexibilización laboral a nivel regional e internacional, para ofrecer un análisis de decisión sobre si es factible o no la flexibilización laboral en nuestro país.

\section{El Desempleo}

En América Latina, a partir del año 2000, se inicia el cambio político regional con la elección del gobierno de Chávez en Venezuela (1998), Lula en Brasil (2001), Kirchner en Argentina (2003), Morales en Bolivia (2005), Correa en Ecuador (2006), Ortega en Nicaragua (2006) o Mujica en Uruguay (2009), que originó cambios en el derecho social. Las raíces de este cambio se dan después de tres décadas marcadas por las dictaduras militares y la imposición del llamado neoliberalismo. Las resistencias populares se manifestaron en las urnas que generaron las condiciones políticas propicias para la llegada al poder de los gobiernos del socialismo del siglo XXI, donde se traducen en políticas laborales y económicas donde no existan imposiciones del Banco Mundial ni del Fondo Monetario Internacional (Gaussens, 2016). En Ecuador específicamente, fueron diez años de cambios profundos, pero no fueron lo suficiente fuertes, para sentar las bases que permita afrontar una nueva crisis. Según Powell la región debería desplegar mas esfuerzos para que se puedan armonizar las reglas y los marcos regularlos que permitan el intercambio de bienes, servicios y suministros para la producción. La integración regional son vías para incrementar la productividad y el crecimiento económico de la región, centrando la atención en políticas para crear empleos de buena calidad, políticas de educación y competencias que favorezcan la empleabilidad y un marco laboral que garantice buenas condiciones de trabajo (CEPAL, 2018b).

La crisis básicamente se ha generado por el desplome del precio del barril de petróleo, ha desencadenado en aumento del nivel de desempleo. La crisis del petróleo a supuesto una crisis estructural. De hecho, normalmente la primera reacción de las empresas ante la caída de sus ventas producida por la crisis, es eliminar las horas extraordinarias y si la situación se plantea más fuerte, llegan a los recortes de personal. En una economía en recesión, es probable que el trabajador continúe cesante por largo tiempo, pues son pocas donde las empresas contratarán personal. En recesión, por lo tanto, es mejor la reducción de la jornada laboral al despido. El contexto actual, aparecen dos incóg- nitas de difícil resolución: no se sabe hasta cuándo va a durar la crisis económica, y qué incidencia presentará la recuperación de la crisis con respecto al desempleo (García, 2015).

El desempleo es un factor importante para la expansión de la pobreza, el desempleo obliga a llevar a cabo un consenso sobre la política económica ejecutada por el gobierno, aunque el desempleo es un problema general de nuestros tiempos, será imposible erradicarlo en su totalidad sin buscar políticas económicas alternativas para mitigarlo, pues el trabajo adecuado y suficientes para todos tiene que convertirse en una aspiración de todo gobierno, pues la economía necesita expandirse en direcciones que creen más trabajo y mejor. (David Sheppard, 1999)

El desempleo mundial al 2018, ha llegado a cifras alrededor de los 190 millones de personas según datos de la OIT y según sus estudios el empleo vulnerable (empleos por cuenta propia) aumentará para los países en desarrollo y emergentes.

Ecuador registró una tasa de desempleo promedio de las 3 principales del Ecuador de $6.5 \%$ en marzo de 2016 en comparación al 3,80 \% que alcanzó en diciembre del 2015, un incremento 2,7 puntos porcentuales, lo que representa una diferencia estadísticamente significativa, según el "Reporte de Economia Laboral “ (INEC, 2018).

Tabla 1 Tasa de Empleo de las tres principales ciudades del Ecuador periodo marzo 2008- marzo 2018

\begin{tabular}{|c|c|c|c|r|}
\hline PERIODO & QUITO & GUAYAQUIL & CUENCA & PROMEDIO \\
\hline mar-08 & 6,5 & 8 & 5 & 6,5 \\
\hline mar-09 & 7,2 & 14,1 & 4,9 & 8,7 \\
\hline mar-10 & 7,2 & 12,3 & 3,8 & 7,8 \\
\hline mar-11 & 5,7 & 10 & 4,1 & 6,6 \\
\hline mar-12 & 3,7 & 6,3 & 4,7 & 4,9 \\
\hline mar-13 & 4,1 & 5,5 & 3,2 & 4,3 \\
\hline mar-14 & 4,3 & 6,1 & 3,2 & 4,5 \\
\hline mar-15 & 4,4 & 3,8 & 3,2 & 3,8 \\
\hline mar-16 & 7,8 & 7,2 & 4,6 & 6,5 \\
\hline mar-17 & 9,1 & 5,1 & 4,3 & 6,2 \\
\hline mar-18 & 7,1 & 4,7 & 5,4 & 5,7 \\
\hline
\end{tabular}

Fuente: Ecuador en cifras

En el mismo informe indica que la tasa de subempleo de las tres ciudades entre marzo 2008 registró: Quito 7,8\%; Guayaquil 16,9\% y Cuenca 9,2\% mientras en Marzo 2018 fue de: Quito 11,3\% Guayaquil 20,2\% y Cuenca 10,2.

Incrementar las oportunidades de trabajo, de un modo distinto al convencional, pero cabal y a escala nacional a través de la flexibilización de empleo, es una vía hacia un rumbo cada vez menos desgastado por la pobreza y la desesperanza, no obstante ninguna flexibilización podría lograr que los aspirantes quieran tener un empleo y lo consigan incluso si es que no se encuentran verdaderamente aptos para ello; por esta razón es imprescindible la capacitación laboral, a través de mecanismos adecuados y efectivos para hacerlo acorde con el desarrollo tecnológico y global. Tanto para lograr un alto nivel de productividad como para enfrentar el acometer con altas y modernas 
técnicas que permitan enfrentar el reto de la competitividad, urge acometer con altas y modernas técnicas que permitan enfrentar nuevos desafíos económicos y empresariales, y hacer más de lo que actualmente se hace. La generación de empleo asalariado sigue siendo insuficiente para absorber el aumento constante de la fuerza laboral (CEPAL, 2018a).

Desde que ingresamos a la era de la información, su característica más importante son los cambios cada vez más rápidos. Vivimos en la época en que la información está en tiempo real, para todos las empresas que sean capaces de recoger la información y transformarla en menos tiempo en un nuevo producto o servicio, antes de que otros lo hagan, tendrán éxito. Así también, el recurso financiero dejó de ser valioso, la economía basada en la manufactura y explotación de los recursos naturales es cambiada por una economía basada en el valor del conocimiento, de la información de la creatividad, de la innovación, siendo importante el conocimiento del capital humano. Es decir, el capital intelectual se ha convertido en el recurso más valioso que tiene la empresa. El trabajo manual ha sido sustituido por el trabajo intelectual, las personas son valoradas por sus conocimientos, sus competencias, sus destrezas y habilidades para la toma de decisiones, y deberán ser consideradas como los nuevos socios del empresario exitoso (Sánchez, 2010).

Las oficinas cerradas e individuales tienden a ser reemplazadas por salas colectivas donde laboran equipos multifuncionales, y las estructuras piramidales se transforman en estructuras planas, hasta llegar a oficinas virtuales, cero papeles, vinculadas electrónicamente, que trabajan mucho más eficientemente estando más cerca del cliente y mejor comunicadas. Es debido a este entramado de situaciones que resulta interesante y apremiante dar un giro al actual tratamiento del recurso humano dentro de las leyes ecuatorianas.

\section{La Flexibilización Laboral}

La flexibilidad en el mercado del trabajo surge en Europa a partir de los 80, producto de las mayores tasas de desempleo observadas en gran parte de los países europeos en comparación con Estados Unidos. , así se extienden los estudios comparativos sobre legislación laboral y su efecto en el mercado laboral. En América Latina nace asociada a los costos sociales de la crisis macroeconómica de los 80. Esto ha llevado a muchos países desarrollados y en vías de desarrollo a la flexibilización laboral, para lograr mayores niveles de empleo y conseguir la adaptación de la fuerza laboral a las cambiantes condiciones económicas (Gonzalez, 2003).

La flexibilización laboral hace alusión, a una visión crítica de las normas jurídicas sobre el trabajo asalariado, que se desarrolla en un aumento de competitividad y que está en el supuesto de ser una receta para mejorar el desempeño empresarial y la creación de empleo, también es una noción desreguladora de normas jurídicas sobre el trabajo y con ello la supresión de derechos como fórmula para el éxito empresarial (Lopez, 2002).

El concepto de flexibilidad, se relaciona con la jornada de trabajo y su extensión, particularmente lo relativo a permitir su flexibilización y extender el periodo de referencia sobre el que se aplican las diversas regulaciones de la normativa laboral. El sistema de trabajo en turnos se asocia a la flexibilidad en el margen intensivo o flexibilidad interna (Ramos, 2003).

La flexibilidad no es una palabra exclusiva para el sector empresarial, sino una cualidad de la sociedad contemporánea que debe poseer, si quiere beneficiarse del cambio y de las oportunidades. La globalización, las nuevas tecnologías de la información y la lucha de las mujeres por lograr la igualdad, están transformando el mercado de trabajo, las medidas destinadas a aumentar el número de puestos de trabajo, la educación en las empresas y las formas de mejorar las condiciones de los trabajadores no son suficientes. No cubren las interacciones desencadenadas por los procesos de cambio producto de la globalización y de la nueva organización del trabajo (Carnoy, 2001).

El debate vuelve a la mesa de discusión, sobre si es conveniente aplicar o no medidas de flexibilización laboral en situaciones de crisis ocupacional, partiendo de una legislación laboral que propicie el empleo mediante la creación de estímulos empresarial para la creación de puestos de trabajo, que rebajen el costo empresarial de la utilización de recurso humano y a su vez permita generar más empleo.

En la época actual las máquinas reemplazaron la energía y el esfuerzo físico del hombre, ahora están reemplazando la paciencia mental y la destreza manual, los ordenadores realizan la tarea de las personas, los trabajadores no cualificados pueden resultar superfluos con el cambio tecnológico. Otro hecho está en que las mujeres esperan formar parte al igual que los hombres en la fuerza productiva, lo están logrando, por eso se requiere adoptar una perspectiva histórica para el momento de legislar con inclusión de todos los sectores (Origlia, 2011).

Para los países de América Latina entre ellos Ecuador y quienes diseñan las reformas laborales para la región, han debido desarrollarla dentro de una época de cambios para los diferentes agentes de la economía: las empresas, los sindicatos, los gremios empresariales y los gobiernos autónomos.

La Constitución de la República del Ecuador del 2008 (Constitución, 2008), establece enfáticamente en sus Artículos 33 y 34 que, es deber del Estado garantizar el trabajo y la seguridad social. La obligación del Estado es la de reestablecer igualdades y velar por que no se profundicen las desigualdades y así como políticas que eviten la inestabilidad laboral.

Hay varias leyes en materia laboral que se deben integrar en un solo cuerpo legal, además de igual número de disposiciones que deberían ser codificados, la propuesta de la reforma laboral en el Ecuador planteada para el 2016, "Proyecto de Ley para la Optimización de la Jornada 
Laboral y Seguro de Desempleo" planteó los siguientes puntos:

El primer lugar establece flexibilizar el empleo para permitir que, en condiciones extremas y con un acuerdo previo entre empleadores y empleados, se pueda reducir la jornada laboral y pagar un salario en relación con las horas trabajadas.

El segundo punto de la reforma laboral consiste en incentivar la contratación de los jóvenes entre 18 y 24 años, el Estado plantea cubrir el aporte a la seguridad social por un salario básico que le correspondería al patrono cuando se contrate a personas de entre 18 y 24 años, sin experiencia laboral previa.

El tercer punto introduce nuevas formas de contratación en el sector de la construcción a través de un contrato a plazo fijo por el tiempo de ejecución de la obra, tal como se hacía antes, cancelando el proporcional de décimos, vacaciones y desahucio de forma mensual. En el sector agropecuario se plantea un contrato por un año renovable pudiendo ser discontinuo; y el sector pesquero, se plantea que la duración del contrato, la jornada laboral y la forma de pago sean acordadas entre las partes.

En cuarto lugar es que el etiquetado de mercancía importada se haga en el país antes de su nacionalización. Lo que le permitirá al país generar nuevas fuentes de empleo.

Otros aspectos que contiene la ley está el aumento de los periodos de lactancia de la mujer que sale del parto, de 3 meses a un año, incluyendo al esposo, algo muy discutible para el sector empresarial por representarle un alto costo.

Por último, está el seguro de desempleo, con el que se busca cambiar la cesantía, que ya tiene la seguridad social y hacer un verdadero plan de pagos mientras la persona está desempleada. (El Comercio, 2016). El desempleo a largo plazo disminuye al existir mayor demanda de trabajo, las fluctuaciones entre el empleo y el desempleo incrementan y disminuyen dependiendo del número de empleados que ingresan y salen del empleo, haciendo que el mercado de trabajo se dinamice.

Por la situación actual de la economía ante las reformas laborales, muchos sectores empresariales coinciden que estas reformas, no son suficientes para mejorar la situación económica del país.

El sector privado aumenta el consumo en periodo de crecimiento produciéndose un aumento del empleo. Por el contrario, el nivel del consumo baja en periodos de recesión dando como consecuencia que se eleven las tasas de desempleo y eso provoque el incremento del empleo no adecuado desplazando al empleo adecuado. Las empresas y todo el sector privado entra en una flexibilidad interna, ya que tiene que desarrollar su capacidad para asignar a los trabajadores a unas u otras tareas dentro de la empresa.

Parte de toda esta problemática es la insuficiencia laboral, que si bien es cierto no se resuelve por medio de reformas legales, no es menos cierto que corresponde crear instituciones que tiendan a mantener el equilibrio entre salario y precios, para lo cual se requiere forzosamente del concurso de los empresarios, los trabajadores y del gobierno, de manera concordante y voluntaria, llámese Dialogo Social Tripartito. El derecho laboral vuelve a ser la base para en el nexo jurídico establecido entre el trabajador y la empresa. Es necesario que desaparezcan las amenazas actuales, como la formación del trabajo no decente en los trabajadores o el desarrollo de la competencia desleal en el sector empresarial (García, 2015).

Es difícil lograr el equilibrio pero es necesario enfrentar esta situación y buscar soluciones; al no afrontar este conflicto de intereses, se da la impresión de que no se lo quiere resolver, en consecuencia se continuará con la contraproducente práctica de aumentar sueldos y salarios cada cierto tiempo, que llevaría a la quiebra de las empresas.

La flexibilización del empleo, va a depender de muchas variables entre ellas el entorno económico mundial, el cambio tecnológico, de la disposición de las empresas a contratar nuevos trabajadores , la política macroeconómica, el clima político, la inversión pública y la inversión privada en capital humano, que son cruciales para configurar en el incremento del número de empleos (Carnoy, 2001).

\section{Estabilidad Laboral}

Las legislaciones del derecho laboral latinoamericano inician su acción al comienzo del siglo pasado, luego viene el nacimiento de la Organización Internacional del Trabajo OIT en 1919. La OIT tiene como una de sus principales actividades fomentar la cooperación entre gobiernos y organizaciones de trabajadores y empleadores en la promoción del progreso social y económico (OIT, 2017). La consolidación del Derecho Laboral en el periodo de entreguerras y en los albores del siglo XX se inicia un proceso sostenido de legislación laboral en todos los países de Latinoamérica: Argentina, Chile , Cuba, México, Guatemala, Uruguay y otros; resulta un antecedente imprescindible nombrar para una aproximación al surgimiento de la legislación del trabajo, su evolución y los paradigmas, tratándose de formas jurídicas, que no tienen un carácter perpetuo, sino que siguen sufriendo transformaciones y desarrollo por los cambios que han tenido las sociedades.

Mario de la Cueva, define al derecho del trabajo como "el conjunto de normas que el pueblo y el poder legislativo ha dado a los trabajadores que están garantizadas en la Constitución y en la Ley del trabajo" (Cueva, 1984), en su obra Mario de la Cueva sostiene que el derecho del trabajo, si bien es un derecho inconcluso, es un derecho imperativo e incuestionablemente protector de la clase trabajadora.

La estabilidad laboral o la garantía de la conservación del empleo mientras no haya causa justa en contrario y la correlativa prohibición del despido, es el derecho del trabajador a permanecer en su puesto de trabajo, incluso contra la voluntad del empresario, mientras no exista causa relevante para ejecutar el despido. En derecho la regla de oro de la estabilidad laboral, es la duración indefinida del contrato trabajo (Pasco, 1989). En el Ecuador el estado reconoce el derecho a la estabilidad laboral. Sin embargo, 
la globalización que trajo consigo la flexibilización laboral también ha provocado la perdida de la estabilidad laboral. Para las empresas la flexibilidad laboral es una salida a una preocupación constante de aumentar la competitividad y productividad, la rigidez de las leyes laborales y la estabilidad laboral del personal que a veces no cumple con las metas, objetivos y expectativas de las empresas hacen que el régimen laboral rígido, se convierta en una constante amenaza (Gomez, 2013).

Uno de los problemas más alarmantes de nuestros tiempos es el crecimiento del sector informal, que en muchos casos lleva a la precariedad del trabajo. No debe coexistir la precarización con la flexibilización. Pero hay conceptos recurrentes: crisis y flexibilidad así como conflicto y concertación social (Melgar, 1989). La estabilidad laboral no puede atentar contra la eficiencia, así como flexibilizar la norma laboral no es desconocer los derechos adquiridos que tienen los trabajadores, ni evadir las responsabilidades patronales. Las perspectivas de crecimiento económico mundial según el Fondo Monetario Internacional, es sobre un crecimiento lento y la expansión del empleo en el sector de servicios de todo el mundo desacelerado (OIT, 2018). Esta desaceleración conlleva a cambios en el trabajo, y merma las posibilidades de encontrar empleo.

\section{Conclusiones}

La difícil situación económica que atraviesa el Ecuador, ha desembocado en un problema de aumento del desempleo.

La productividad de una empresa y la competitividad del país depende del buen funcionamiento del mercado laboral, de ahí la importancia de una legislación laboral moderna, concreta, novedosa y potente, que concilie la flexibilidad y la estabilidad del trabajador.

Es importante un consenso entre empleadores y empleados, a patronos y trabajadores de todos los sectores de la economía, a los especialistas en las diferentes ramas para ofrecer medidas claras y duraderas, medidas que realmente se mantengan en el tiempo en beneficio de los ecuatorianos, medidas que realmente aumenten el empleo producto de la producción y el mejoramiento de la productividad.

Es necesario la concertación popular y empresarial para que junto al Estado se analice todas las medidas, para fomentar la creación de empleos y los aspectos que benefician a todos, por eso es necesario el diálogo, trabajando en un unísono para beneficio de los ecuatorianos.

\section{Referencias Bibliográficas}

Carnoy, M. (2001). El Trabajo Flexible en La era De La Informacion. Descargado de https://goo.gl/ PXRgjY

CEPAL. (2018a). Estudio Económico de America Lativa y el Caribe (Inf. Téc.).

CEPAL. (2018b). Perspectivas economicas de America Latina (Inf. Téc.). Paris.
Constitución. (2008). Constitución.

Cueva, M. (1984). El nuevo derecho mexicano del trabajo. En (p. 96). México: Porrúa.

David Sheppard, R. D.-S. (1999). El Desempleo y el futuro del trabajo: una investigación para las Iglesias. Bilbao: Editorial Santander.

El Comercio. (2016). El Comercio. Descargado de https://goo.gl/6intRt

García, J. (2015). Economía Sumergida y relaciones laborales en Europa. Revista Latinoamericana de Derecho Social, 37.

Gaussens, P. (2016). ¿El Fin del Trabajo o el trabajo como fin? Revista Latinoamericana de Derecho Social, 34 y 35 .

Gomez, M. A. (2013). No Title. Revista Diversitas, 10(1).

Gonzalez, P. (2003). Politica de Empleo e Institucionalidad Laboral para el siglo XXI. En (p. 73). Santiago de Chile: Editorial Universitaria.

INEC. (2018). Reporte de Economia Laboral (Inf. Téc.). Quito.

Lopez, D. (2002). Mitos, Alcances y Perspectivas de la flexibilizacón laboral, un debate permanente. Análisis Laboral, 1,.

Melgar, A. M. (1989). Estabilidad en el empleo, solución de conflictos de trabajo y concertación. Murcia: Selegráfica.

OIT. (2017). No Title. Descargado de https://goo . $91 / \mathrm{nkQP} J \mathrm{~J}$

OIT. (2018). Perspectivas Sociales y del Empleo en el Mundo (Inf. Téc.). Ginebra.

Origlia, G. (2011). Paso firme hacia la equidad de género. Estrategia y Negocios, 2.

Pasco, M. (1989). Estabilidad en el empleo, solución de conflictos de trabajo y concertación social. En (p. 39). Murcia: Selegráfica.

Ramos, J. (2003). Politicas de empleo e institucionalidad laboral para el siglo XXI. Santiago de Chile: Editorial Universitaria.

Sánchez, F. (2010). El valor de la capacitación personalizada en las empresas. Descargado de https:// goo.gl/YkBPY2

Recibido: 10 de agosto de 2018

Aceptado: 15 de octubre de 2018 
